\title{
An update on activities of the International Society for Child and Adolescent Injury Prevention
}

As I write this, it has been two years since the International Society for Child and Adolescent Injury Prevention was born at the Second World Conference on Injury Control. Membership has slowly grown and is now over 100 . We have mailed out many membership application forms, and have provided applications at recent injury control conferences. The Society is our organization; its up to us to talk about it to others and to recruit new members. Publication of the journal has helped tremendously in membership recruitment and mailings have produced new members as well. Word of mouth from current members of ISCAIP, however, is the most effective way of expanding our membership. So let's all help! Each one recruit one.

A number of new society activities have taken place in the last few months. We now have a part time staff person, Angela Seay, working out of the Child Accident Prevention Trust (CAPT) office in London, to help with recruitment, raising funds, and keeping track of activities. She can be reached through CAPT or by e-mail at 100545.3625@compuserve.com.We are very pleased to have her working with us.

We have begun to plan the meeting for the society at the Third International Conference on Injury Control in Melbourne next February. The Society, along with ChildSafe of Australia, will host a meeting on Thursday afternoon, immediately following the closing of the main conference. This will allow us to have a large enough block of time to devote to our topics. We plan to have speakers provide an update on recent advances in child and adolescent injury control in the Americas, Europe, Australia, New Zealand, Asia, and Africa. Another part of the session will be devoted to discussing current challenges in injury control for transport, home/leisure, intentional and occupational injuries as well as injuries in rural areas and developing countries. The focus will be on the next steps that need to be taken to further decrease injuries in these areas.

Finally, I would like us to have an open discussion on how ISCAIP can best service its members and make an impact on injury control in countries around the world. We will have a business meeting as well to elect board members and officers.

Please look for information about the meeting in future mailings and plan on coming. It will be open to all.

We are presently trying to obtain funds for the two ISCAIP sponsored projects. The first is a survey of child and adolescent injury control activities around the world, combined with a collection of up-to-date statistics on injury mortality. Much can be learned from a comparison of injury rates and programs that have been instituted to lower those rates. Our hope is to secure this funding and to present the results of the survey at the February, 1996 meeting.

The second project is a systematic review and metaanalysis of all existing randomized controlled trials of interventions to prevent injuries to children and adolescents. Finding out what works in injury control is of tremendous public health importance. Systematic literature reviews and meta-analyses are invaluable methods of synthesizing the existing evidence from randomized controlled trials. Health care providers, policy makers, and injury control professionals are faced with large amounts of information, distributed through a large number of sources. This requires systematic reviews to integrate the diverse information and thus provide a basis for rational decision making. It is quite likely that when currently available evidence on the efficacy of injury prevention interventions is thoroughly synthesized, many interventions believed to be effective will be shown to be ineffective and vice versa. In addition, systematic reviews are likely to show that some proposals for future research are redundant because intervention efficacy can already be established from existing evidence. Most importantly, such reviews will clarify which programs are appropriate to implement on a broader scale.

This project would be part of the Cochrane Collaboration, an international network of individuals and institutions that has been developed to conduct systematic reviews of health care interventions. A literature search will be conducted to find the studies, and then individual members of ISCAIP will be called on to conduct a review of these studies. These will be combined using meta-analysis to evaluate the net impact of different types of interventions on various injury control problems in the pediatric age group. Funds are currently being sought to support this undertaking.

Injury Prevention is the official journal of the Society. Let us know how well it is serving your needs. Do you like the content, format, and layout of the journal? What can we do better? The journal is still in its infancy and like all young children it needs guidance and shaping as it grows and develops.

FREDERICK P RIVARA

Harborview Injury Prevention and Research Center, Chair, ISCAIP

Box 359960, 325 Ninth Avenue,

Seattle,

WA 98104, USA

(Phone: +1 206521 1530, fax: + 1206521 1562, e-mail: fpr@u. washington.edu 